\title{
DIAGNOSTIC ACCURACY OF PERFUSION WEIGHTED MRI IN DIFFERENTIATING NEOPLASTIC AND NON-NEOPLASTIC BRAIN LESIONS USING HISTOPATHOLOGY AS GOLD STANDARD.
}

1. MBBS, FCPS, FRCP

Fellowship of Intervention Radiology

AKU, Karachi.

Senior Registrar

Allied Hospital, Faisalabad.

2. MBBS

Medical Officer

Department of Radiology

Allied Hospital, Faisalabad.

3. MBBS

House Officer

Allied Hospital, Faisalabad.

4. MBBS

Medical Officer

Allied hospital Faisalabad.

Correspondence Address:

Dr. Muhammad Ahsan

Allied hospital Faisalabad.

ahsanjahangir194@gmail.com

Article received on:

14/12/2018

Accepted for publication:

22/03/2019

Received after proof reading: 30/09/2019

\begin{abstract}
Abdul Raouf', Anem Mahboob², Tajawar Gul ${ }^{3}$, Muhammad Ahsan ${ }^{4}$
\end{abstract}
ABSTRACT... Objectives: Perfusion-weighted imaging may be performed as a complementary examination to conventional MRI techniques. The clinical applications of PWI in the evaluation of focal brain lesions include the differentiation between neoplastic and non-neoplastic brain lesions, primary tumors and solitary metastasis and, in the post-treatment follow up, the differentiation of tumoral recurrence and radio necrosis. To determine the diagnostic accuracy of perfusion weighted MRI in differentiating. Neoplastic and non-neoplastic brain lesions keeping histopathology as gold standard. Study Design: Cross sectional (validation) study. Setting: The study was conducted in Radiology department of Allied Hospital Faisalabad. Period: Six months from 25-March-2016 to 24-Sep-2016. Material and Methods: Permission for this study was taken from the hospital ethical review committee. A total of 125 patients were included and MRI examination was performed with the patients in supine position using a 1.5-T Philips MRI unit and a body phased-array coil. A preload of paramagnetic contrast agent (gadolinium) was administered 30 seconds before acquisition of dynamic images, followed by a standard dose 10 seconds after starting imaging acquisitions. Results: Patients ranged between 15-65 years of age. Mean age of the patients was $47.6 \pm 10.6$ years. There were 65 males $(52 \%)$ and 60 females (48\%). PW MRI showed sensitivity $81.67 \%$, specificity $81.54 \%$, positive predictive value $80.33 \%$, negative predictive value $82.81 \%$ and diagnostic accuracy $81.60 \%$. ROC and likelihood ratio was measured for age and gender. For age (15-40 years) likelihood ratio was 7.187 and for age 41-65 likelihood ratio was 48.665. For males likelihood ratio was 31.759 while for females 16.188. Conclusion: In conclusion, our results suggest a promising role for perfusion MR imaging in the distinction between neoplastic and non-neoplastic lesions.

Key words: $\quad$ Neoplastic Brain Lesions, Non-Neoplastic Brain Lesions, PW-MRI.

Article Citation: Raouf A, Mahboob T, Ahsan M. Diagnostic accuracy of perfusion weighted $\mathrm{MRI}$ in differentiating neoplastic and non-neoplastic brain lesions keeping histopathology as gold standard. Professional Med J 2019; 26(10):16251629. DOI: $10.29309 / T P M J / 2019.26 .10 .2471$

\section{INTRODUCTION}

It is a difficult task for physicians and surgeons to differentiate neoplastic and non-neoplastic brain space occupying lesions (SOL) ${ }^{1,2}$ Simple non-invasive imaging techniques cannot provide detailed dimensions of the SOL; because of this reason many patients end-up into invasive pathological testing. ${ }^{1}$

\section{Contrast enhanced Magnetic resonance} imaging (MRI) is the current standard technique for neurosurgeons in diagnosis of brain lesions. ${ }^{3,4}$ But still, simple MRI techniques are limited in the assessment of size, site and shape of the lesion, along with the stage of the malignant disease while differentiating tumor recurrence from radio-necrosis. ${ }^{3}$ In the past few decades much advancement have been made in the MR protocols thus improving the assessment of functional tissue parameters like blood supply or metabolic process. The diagnosis of various brain diseases along with the post-management -related changes can be easily seen by the improved and advanced techniques. ${ }^{5}$

Perfusion-weighted imaging (PWI) is one of the advanced techniques used by neuro-radiologist. Brain PWI is done along with plain MRI i.e the conventional technique, in order to access the brain parameters and mapping of the blood microcirculation. ${ }^{3}$ Our aim was to differentiate to employ to distinguish between infectious 
and neoplastic brain lesions by observing brain microcirculation changes. ${ }^{6}$ Microscopy PWI also provides reliable biomarkers for glioma grading, therapeutic responses, and differential diagnosis of various brain tumours. ${ }^{7}$ PW MRI is also helpful in diagnosis of sellaar/parasellar SOLs; thus it can beuse in the diagnosis of all intracranial lesions as it is a faster technique. ${ }^{8}$

Wrong diagnosis or wrong estimation of the SOL anatomy can lead to treatment-delay or even over-treatment. ${ }^{9}$ The sensitivity of PW MRI for the diagnosis of neoplastic and non-neoplastic SOL is $87.2 \%$ and specificity of $70.6 \%^{10}$ as compared to histopathology, the gold standard method of investigation, which has sensitivity and specificity of almost $100 \% .{ }^{11}$ Prevalence rate of neoplastic lesions is $54 \%$ and of nonneoplastic lesions is $46 \%{ }^{3}$

Therationale ofmystudy is to assess theusefulness of Perfusion Weighted MRI in distinguishing between focal neoplastic and infectious brain lesions according to histopathology. Misdiagnosis leading to unwarranted neurosurgery or exposure to toxic chemotherapy can be avoided by this technique. This study will help both patients as well as doctors by providing accurate diagnosis of the focal brain lesions which would help opting for targeted treatment plans.

\section{MATERIAL AND METHODS}

This cross sectional (validation) study was conducted in the Radiology department of Allied and DHQ hospital Faisalabad six months after approval from the hospital ethical review committee. Patients under the age of 15 and 65 including both genders (males and females) having diagnosis/suspicion of space occupying lesion in brain on CT scan were included in the study.

Neoplastic Lesions were defined as lesions that have higher rCBV levels on PW MRI are considered as focal neoplastic lesions. Among them, low grade gliomas have lower rCBV value than high grade gliomas but more than nonneoplastic lesions. According to histopathology, neoplastic lesion may show an increase in the size of the nucleus of the cells or the cell as a whole, anaplasia or hyperchromatism or prominent nucleoli or irregular chomatin in the nucleus. They can potentially invade and metastize.

Non-neoplastic SOL was defined as any lesion with low rCBV value along with edema of the periphery of lesion. According to histopathology, non-neoplastic lesions are slow growing, resembles to tissue of origin (well differentiated), Circumscribed, non-invasive and do not metastize. Perfusion Weighted MRI Criteria for Differentiating Neoplastic and Non-neoplastic Brain Lesions were defined as PW MRI showed that the rCBV value of neoplastic lesion was found to be significantly higher than that of non-neoplastic lesion, with an optimal cut-off rCBV value of 1.3 3 permitted distinction with high sensitivity and specificity. Sensitivity was defined as the ability of Perfusion Weighted MRI to detect those who have neoplastic brain lesion. Specificity was defined as the ability of Perfusion Weighted MRI to exclude those who don't have neoplastic brain lesion. True positive was defined as the cases that will be positive for neoplastic brain lesion on Perfusion Weighted MRI and histopathology. True negative was defined as the cases that will be negative for neoplastic brain lesion on Perfusion Weighted MRI and histopathology. False-positive were defined as the cases that will be positive for neoplastic brain lesion on PW MRI but negative on histopathology. False-negative was defined as the cases that will be negative for neoplastic brain lesion on PW MRI but positive on histopathology. Positive predictive value (PPV) was the probability of having neoplastic brain lesion if found +ve on Perfusion Weighted MRI. Negative predictive value was defined as the probability of not having neoplastic brain lesion if found -ve on Perfusion Weighted MRI.

Patients undergoing chemotherapy, irritable patients, patients who are claustrophobic or have a history of claustrophobia and patients with history of trauma or surgery leaving behind any magnetic implants or pacemakers, were excluded from the study.

Sample size if 125 were collected by using WHO 
sample size calculator with sensitivity level of $87.2 \%^{12}$ and specificity level of $70.6 \%{ }^{12}$ with prevalence rate of $54 \%{ }^{3}$ confidence level of $95 \%$ with absolute precision of $10 \%$.

MR examination was done while keeping the patients in supine position using a 1.5-T Philips MRI unit and a body phased-array coil. Gadolinium was given over 30 seconds before acquisition of dynamic images, followed by a dose over 10 seconds. rCBV values were calculated using regional cerebral blood volume in the SOL relative to normal white matter of opposite side. The routine PW MRI protocol consisted of sagittal T1-W before and after administration of paramagnetic contrast agent $(\mathrm{TR} / \mathrm{TE}=529 / 15 \mathrm{~ms}$; slice thickness, $5 \mathrm{~mm}$; slice interval, $1 \mathrm{~mm}$; FOV, $250685 \mathrm{~mm}$; matrix, 2566512; NSA $=2$ ), coronal T1-W after administration of paramagnetic contrast agent (TR/ TE $=522 / 15 \mathrm{~ms}$; slice thickness, $5 \mathrm{~mm}$; slice interval, $1 \mathrm{~mm}$; FOV, $190690 \mathrm{~mm}$; matrix, 2246512; NSA $=2$ ) and axial T1-W images with use of magnetization transfer after administration of paramagnetic contrast agent (TR/TE = 435/15 ms; slice thickness, $5 \mathrm{~mm}$; slice interval, $1 \mathrm{~mm}$; FOV, $250670 \mathrm{~mm}$; matrix, 2566512; NSA = 2). Histopathology sample was sent to hospital lab and verified by pathologist. This procedure was performed in Radiology department of Allied hospital and reported by a Senior Radiologist. Data were collected on a predesigned proforma.

Data was analyzed by using SPSS V-16. Descriptive statistics were calculated for all variables. Mean and standard deviation was calculated for all quantitative variables including age. Frequency and percentage was calculated for qualitative variable including true positive and gender. Sensitivity and specificity was calculated using $2 \times 2$ table:

\begin{tabular}{|l|l|l|l|}
\hline PW MRI & \multicolumn{2}{|c|}{$\begin{array}{c}\text { Histopathology } \\
\text { (Gold Standard) }\end{array}$} & Total \\
\cline { 2 - 3 } & Neoplastic & Non-Neoplastic & \\
\hline Positive & TP & FP & \\
\hline Negative & FN & TN & \\
\hline Total & & & \\
\hline
\end{tabular}

\section{RESULTS}

Patients meeting the inclusion criteria were included in the study during the study period of six months from 25-03-2016 to 24-09-2016. Patients ranged between 15-65 years of age. Mean age of the patients was $47.6 \pm 10.6$ years Table-I. There were 65 males (52\%) and 60 females (48\%). PW MRI showed sensitivity $81.67 \%$, specificity $81.54 \%$, positive predictive value $80.33 \%$, negative predictive value $82.81 \%$ and diagnostic accuracy $81.60 \%$. ROC and likelihood ratio was measured for age and gender. Gender distribution shows that $52 \%(n=65)$ were male while $48 \%(n=60)$ were females. (Table-II) and comparison of PW MRI findings vs histapology is shown in Table-III.

\begin{tabular}{|l|c|c|}
\hline \multicolumn{1}{|c|}{ Age } & No. of Patients & $\%$ \\
\hline $15-40$ & 27 & 21.6 \\
\hline $41-65$ & 98 & 78.4 \\
\hline Total & 125 & 100.0 \\
\hline Mean+ SD & \multicolumn{2}{|c|}{$47.6 \pm 6.60$} \\
\hline
\end{tabular}

Table-I. Age distribution $(n=125)$

\begin{tabular}{|l|c|c|}
\hline \multicolumn{1}{|c|}{ Gender } & No. of Patients & $\%$ \\
\hline Male & 65 & 52 \\
\hline Female & 60 & 48 \\
\hline Total & 125 & 100 \\
\hline
\end{tabular}

Table-II. Gender distribution $(n=125)$

\begin{tabular}{|c|c|c|c|}
\hline \multirow{2}{*}{ PW MRI } & \multicolumn{2}{|c|}{$\begin{array}{l}\text { Histopathology } \\
\text { (Gold Standard }\end{array}$} & \multirow{2}{*}{ Total } \\
\hline & Neoplastic & $\begin{array}{c}\text { Non- } \\
\text { neoplastic }\end{array}$ & \\
\hline Neoplastic & $\begin{array}{c}49(\mathrm{TP}) \\
\mathrm{a}\end{array}$ & $\begin{array}{c}12(\mathrm{FP}) \\
b\end{array}$ & 61 \\
\hline Non-neoplastic & $\begin{array}{c}11(\mathrm{FN}) \\
\mathrm{C}\end{array}$ & $\begin{array}{c}53(\mathrm{TN}) \\
\mathrm{d}\end{array}$ & 64 \\
\hline Total & $\begin{array}{c}60 \\
a+c\end{array}$ & $\begin{array}{c}65 \\
b+d\end{array}$ & 125 \\
\hline
\end{tabular}

Sensitivity: $a / a+c \times 100 \quad 81.67 \%$

Specificity: $d / d+b \times 100 \quad 81.54 \%$

Positive Predictive Value $a / a+b \times 100$

$80.33 \%$

Negative Predictive Value: $d / c+d \times 100$

$82.81 \%$

Diagnostic accuracy: $a+d / a+d+b+c \times 100$ $81.60 \%$ 


\section{DISCUSSION}

Magnetic resonance imaging (MRI) is the ideal brain study; but simple MRI has show limited parameters $^{13}$ including difficulty in estimating accurate margin of the SOL, stage and grade of neoplasm with poor differentiation of tumor recurrence and radionecrosis. ${ }^{14}$ Many new complementary MR techniques have been developed including PWI. ${ }^{15}$

Perfusion-weighted imaging (PWI) techniques have made their place in diagnostic radiology. Perfusion-weighted imaging is done along with old MRI techniques for mapping of the. ${ }^{16}$ Cerebral perfusion is the amount of blood supplied to the tissue mass in a second. ${ }^{17}$ However, in PWI, other parameters like cerebral blood volume, cerebral blood flow and the mean transit time. ${ }^{18,19} \mathrm{PWI}$ has role in the diagnosis of focal brain lesions, simple or metastatic disease and differentiation of radionecrosis. ${ }^{20}$

In current study, perfusion weighted MRI in diagnosis of neoplastic brain SOLs revealed sensitivity $81.6 \%$, specificity $81.54 \%$, positive predictive value (PPV) $80.33 \%$, negative predictive value (NPV) $82.81 \%$ with a diagnostic accuracy $81.60 \%$. Our findings are consistent with results of Toh et al. ${ }^{10}$ They demonstrated sensitivity of $\mathrm{PW} \mathrm{MRI}$ in differentiating is $87.2 \% .^{10}$

\section{CONCLUSION}

In conclusion, our results were in favor of use of perfusion MR imaging in the differentiation and diagnosis of various SOLs. An investigation like perfusion MRI, which is non-invasive, and accurately differentiates and categorize neoplastic SOL in brain can prevent biopsies and help in the proper treatment ending up in shorter hospital stay, morbidity and mortality.

\section{CONFLICT OF INTEREST}

The authors of this article declared no conflict of interest.

$$
\text { Copyright } \odot 22 \text { March, } 2019 .
$$

\section{REFERENCES}

1. Nekooei $S$, Haratizadeh $B$. The role of magnetic resonance spectroscopy in differentiation between neoplastic and non-neoplastic focal brain lesions Rev Clin Med 2014; 1:17-20.

2. Alam MS, Sajjad Z, Hafeez S, Akhter W. Magnetic resonance spectroscopy in focal brain lesions. J Pak Med Assoc 2011; 61:540-3.

3. Floriano VH, Ferraz-Filho JRL, Spotti AR, Tognola WA. Perfusion-weighted magnetic resonance imaging in the evaluation of focal neoplastic and infectious brain lesions: Results from a cohort of 100 consecutive patients. Rev Bras Neurol 2010; 46:29-36.

4. Wang W, Hu Y, Lu P, Li Y, Chen Y, Tian M, et al. Evaluation of the diagnostic performance of magnetic resonance spectroscopy in brain tumors: A systematic review and meta-Analysis. PLoS One 2014; 9:e112577.

5. Essig M, Shiroishi MS, Nguyen TB, Saake M, Provenzale JM, Enterline D, et al. Perfusion MRI: the five most frequently asked technical questions. AJR Am J Roentgenol 2013; 200:24-34.

6. Floriano VH, Torres US, Spotti AR, Ferraz-Filho JRL, Tognola WA. The role of dynamic susceptibility contrast-enhanced perfusion MR imaging in differentiating between infectious and neoplastic focal brain lesions: Results from a cohort of 100 consecutive patients. PLoS One 2013; 8;e81509.

7. Bladowska J, Zimny A, Guziński M, Hałoń A, Tabakow $P, C z y z M$, et al. Usefulness of perfusion weighted magnetic resonance imaging with signal intensity curves analysis in the differential diagnosis of sellar and parasellar tumors: Preliminary report. Eur $\mathrm{J}$ Radiol 2013; 82:1292-8.

8. Garg RK, Sinha MK. Multiple ring-enhancing lesions of the brain. J Postgrad 2010; 56:307-16.

9. Lee SK. Diffusion tensor and perfusion imaging of brain tumors in high-field MR. Neuroimaging Clin N Am. 2012; 22:123-4.

10. Toh $\mathrm{CH}$, Wei $\mathrm{KC}$, Chang $\mathrm{CN}, \mathrm{Ng} \mathrm{SH}$, Wong HF, Lin CP. Differentiation of brain abscess from glioblastoma multiforme and metastatic brain tumors: comparisons of diagnostic performance of dynamic susceptibility contrast enhanced perfusion MR imaging before and after mathematic contrast leakage correction. PLoS One. 2014; 9:e109172. 
11. Venneti S, Santi M, Felicella MM, Yarilin D, Phillips JJ, Sullivan LM, et al. A sensitive and specific histopathologic prognostic marker for H3F3A K27M mutant pediatric glioblastomas. Acta Neuropathol $2014 ; 128: 743-53$.

12. Lee AG, Goldberg MF, Gillard JH, Barker PB, Bryan RN. Intracranial assessment of incontinentia pigmenti using magnetic resonance imaging, angiography, and spectroscopic imaging. Arch Pediatr Adolesc Med 1995; 149:573-80.

13. Rollin N, Guyotat J, Streichenberger N, Honnorat J, Tran Minh VA, Cotton F. Clinical relevance of diffusion and perfusion magnetic resonance imaging in assessing intraaxial brain tumors. Neuroradiology 2006; 48:1509.

14. Covarrubias DJ, Rosen BR, Lev MH. Dynamic magnetic resonance perfusion imaging of brain tumors. Oncologist 2004; 9:528-37.

15. Law M, Yang S, Babb JS. Comparison of cerebral blood volume and vascular permeability from dynamic susceptibility contrast enhanced perfusion MR imaging with glioma grade. AJNR Am J Neuroradiol 2003; 25:746-55.
16. Schmainda KM, Rand SD, Joseph AM. Characterization of a first-pass gradient-echo spin-echo method to predict brain tumor grade and angiogenesis. AJNR Am J Neuroradiol 2004; 25: 1524-32.

17. Keston P, Murray AD, Jackson A. Cerebral perfusion imaging using contrast-enhanced MRI. Clin Radiol 2003; 58:505-13.

18. Cha S, Knopp EA, Johnson G, Wetzel SG, Litt AW, Zagzag D. Intracranial mass lesions: dynamic contrast-enhanced susceptibility-weighted echoplanar perfusion MR imaging. Radiology 2002; 223:11-29.

19. Petrella JR, Provenzale JM. MR perfusion imaging of the brain: Techniques and applications. AJR Am J Roentgenol 2000; 175:207-19.

20. Wong JC, Provenzale JM, Petrella JR. Perfusion MR imaging of brain neoplasms. AJR Am J Roentgenol 2000; 174:1147-57.

\begin{tabular}{|c|c|c|c|}
\hline \multicolumn{4}{|c|}{ AUTHORSHIP AND CONTRIBUTION DECLARATION } \\
\hline Sr. \# & Author-s Full Name & Contribution to the paper & Author's Signature \\
\hline 1 & Abdul Raouf & Data collection. & \\
\hline 2 & Anem Mahboob & Data collection \& Data analysis. & \\
\hline 3 & Tajawar Gul & Data collection. & \\
\hline 4 & Muhammad Ahsan & Paper writing, Data analysis. & \\
\hline
\end{tabular}

